Die zahlreichen Beiträge von Bondy im Monat drehten sich vor allem um die Themen Intellektuelle, ${ }^{344}$ Kommunismus, ${ }^{345}$ Nationalsozialismus, ${ }^{346}$ Geschichte $^{347}$ und Literatur $^{348}$. Zudem standen die »westeuropäischen Länder« im Mittelpunkt der Veröffentlichungen. ${ }^{349}$

\title{
4.6 Raymond Aron
}

Eine von 1930 bis Mitte des Jahres 1933 dauernde Tätigkeit als Lektor an der Universität in Köln und anschließend in Berlin wurde für Raymond Aron zu einem intellektuellen und politischen Schlüsselerlebnis. Einerseits studierte er intensiv die deutschen Philosophen Marx, Husserl und Heidegger sowie die Schriften des Soziologen Max Webers, andererseits erlebte er den Aufstieg und die »nationale Revolution « des Nationalsozialismus. Nicht zuletzt der gewalttätige »Antisemitismus der Hitleranhänger« schockierte Aron. ${ }^{350}$ In seiner Autobiografie berichtet Aron davon, wie er zusammen mit seinem Freund Golo Mann am 10. Mai 1933 in Berlin die Bücherverbrennung durch Goebbels und die SA erlebte. ${ }^{351}$

wurde am 14. März 1905 als dritter Sohn assimilierter jüdischer Eltern in Paris geboren. Der Vater war Professor der Rechte. Die Familie zählte zur bürgerlichen Mittelschicht der französischen Juden und stammte ursprünglich aus Lothringen. Der Großvater, ein "glühender Patriot«, und sein Vater wurden durch die Dreyfusaffäre

344 Siehe bes.: Der Zeuge des Verrats, in: Der Monat 4 (1952), H. 48, S. 653-657 (Rezension); Rebellen als Musterschüler. Zu zwei neuen Essaybänden, in: Der Monat 5 (1953), H. 58, S. 407-411 (Sammelrezension); Gentleman und Streiter. Zum Tode George Orwells, in: Der Monat 2 (1950), H. 18, S. 565-567; George Orwell oder Common Sense als Paradox, in: Der Monat 6 (1954), H. 70, S. 396-401; Der Aufstand als Maß und als Mythos. Ein Blick auf das Werk von Albert Camus aus Anlaß von »L'Homme révolté«, in: Der Monat 5 (1953), H. 61, S. 87-96; Albert Camus zum Gedenken, in: Der Monat 12 (1960), H. 137, S. 7 f.

345 Siehe bes. François Bondy, Der expropriierte Marxismus«, in: Der Monat 2 (1950), H. 21, S. 309-313 (Rezension).

346 Siehe: Karl Jaspers zum Eichmann-Prozeß (Ein Gespräch mit François Bondy), in: Der Monat 13 (1961), H. 152, S. 15-19; »]a, mein lieber guter Storfer ...«. Eichmann wörtlich, in: Der Monat 13 (1961), H. 153, S. 58-61.

347 Siehe z. B. die Rezension von J. R. von Salis, Weltgeschichte der neuesten Zeit, Bd. I (1871-1904), Zürich o. J. unter dem Titel Aus nächster Ferne. Zu J. R. von Salis' neuer Weltgeschichte, in: Der Monat 5 (1953), H. 52, S. 433-439, sowie J. R. von Salis, Weltgeschichte der neuesten Zeit, Bd. II (1905-1918) unter dem Titel Der europäische Bürgerkrieg. Zu J. R. von Salis »Weltgeschichte der neuesten Zeit«, in: Der Monat 8 (1956), H. 90, S. 60-62.

348 Siehe Albert Camus' Meisterwerk. Zu seiner Erzählung »La Chute«, in: Der Monat 8 (1956), H. 94, S. 67-71 (Rezension), sowie Die Mandarine des Linken Ufers, in: Der Monat 7 (1955), H. 76, S. 371-374 (Rezension).

349 Siehe z. B. zu Frankreich: De Gaulle zwischen Aufständigen und Aufsässigen, in: Der Monat 13 (1960), H. 146, S. 12-21; zu Italien: Italien ohne De Gasperi, in: Der Monat 7 (1954), H. 73, S. 82-84; zu Spanien: Brief aus Madrid: Was kommt nach Spanien, in: Der Monat 8 (1956), H. 93, S. 33-40; Brief aus Madrid: Die verbrauchte Diktatur, in: Der Monat 9 (1957), H. 104, S. 39-43; Brief aus Madrid: Die Toten und die Lebenden, in: Der Monat 11 (1959), H. 127, S. 3-8.

350 Raymond Aron, Der engagierte Beobachter, Gespräche mit Jean-Louis Missika und Dominique Wolton, Stuttgart 1983, S. $26 \mathrm{f}$.

351 Ders., Erkenntnis und Verantwortung. Lebenserinnerungen, München 1985, S. 54. 
um die Jahrhundertwende tief erschüttert. ${ }^{352}$ Nach dem Besuch des Gymnasiums studierte Aron von 1924 bis 1928 klassische Philosophie an der Eliteschule École Normale Supérieure. $\mathrm{Zu}$ seinen Studienfreunden und intellektuellen Diskussionspartnern zählten Jean-Paul Sartre und Paul Nizan. Als Jahrgangsbester beendete er sein Studium mit einer Diplomarbeit über die Philosophie Kants, die ihn befähigte, eine Tätigkeit als Philosophielehrer der gymnasialen Oberstufe (Agrégation) auszuüben. ${ }^{353}$ Sein damaliges Selbstverständnis eines politischen Linken ließen ihn Mitte der 1920er-Jahre Mitglied in der Sozialistischen Partei werden. Während seines Deutschlandaufenthaltes erfolgte neben dem Studium der phänomenologischen Schriften Husserls und Heideggers eine ausführliche Lektüre der ökonomisch-philosophischen Werke von Marx, die ihn, wie auch die intellektuelle Entdeckung Webers, für die zukünftige Auseinandersetzung mit dem Totalitarismusphänomen entscheidend beeinflussen sollte.

Nach seiner Rückkehr aus Deutschland beschäftigte sich Aron mit genuin wissenschaftlichen Arbeiten zur Soziologie, Philosophie und Geschichte. Neben der Fertigstellung seiner Habilitationsschrift Introduction à la philosophie de l'histoire 1938, die eine Absage an jedwede geschichtsphilosophischen Vorstellungen von einem universellen Determinismus der geschichtlichen Ereignisse enthielt, ${ }^{354}$ schrieb Aron auch und vor allem über die neuartigen Herrschaftssysteme in Deutschland, Italien und ab Ende der 1930er-Jahre über die in der Sowjetunion. ${ }^{355}$ Die Wahlergebnisse, den Aufstieg und die »Machtergreifung « der NS-Massenbewegung sowie die Politik der konservativen Elite in Deutschland ${ }^{356}$ nahm der »engagierte Beobachter« Aron als Gefahr für Frank-

352 Ebd., S. 14.

353 Brigitte Gess, Raymond Aron, in: Karl Graf Ballestrem/Henning Ottmann (Hg.), Politische Philosophie des 20. Jahrhunderts, München 1990, S. 33-45, hier S. 34. Von der Autorin ist eine der wenigen grundsätzlichen wissenschaftlichen Arbeiten über Aron in Deutschland erschienen. Siehe Brigitte Gess, Liberales Denken und intellektuelles Engagement. Die Grundzüge der philosophisch-politischen Reflexionen Raymond Arons, München 1988. Vgl. zu Aron auch Harald Seubert, Erinnerungen an den >Engagierten Beobachter in veränderter Zeit. Über Raymond Aron als Theoretiker des Totalitarismus und der nuklearen Weltlage, in: Hans Maier/Michael Schäfer (Hg.), »Totalitarismus« und »politische Religionen«. Konzepte des Diktaturvergleichs, Bd. 2, Paderborn u. a. 1997, S. 311-361.

354 Siehe im Einzelnen hierzu Joachim Stark, Das unvollendete Abenteuer. Geschichte, Gesellschaft und Politik im Werk Raymond Arons, Würzburg 1986, S. 52-133 (hier befindet sich im Anhang eine umfangreiche Bibliografie der Schriften Arons). Zur Habilitationsschrift im Speziellen siehe Brigitte Gess, Raymond Aron, S. 35 ff. Nach seiner Rückkehr aus Deutschland nach Frankreich im Laufe des Jahres 1933 und einer vorübergehenden Tätigkeit als Gymnasiallehrer in Le Havre und als Universitätsprofessor in Saint-Cloud von 1935 bis 1939 sowie 1939 an der Universität Toulouse war Raymond Aron parallel hierzu von 1938 bis 1939 Sekretär des Zentrums für soziale Dokumentation an der Pariser École Normale Supérieure.

355 Diese Texte, die ursprünglich für verschiedene Monatszeitschriften in Paris geschrieben wurden, sind enthalten in: Joachim Stark (Hg.), Raymond Aron: Über Deutschland und den Nationalsozialismus. Frühe politische Schriften 1930-1939, Opladen 1993 (in dem Band sind auch die entsprechenden Angaben zum Ort und Zeitpunkt der jeweiligen Veröffentlichungen enthalten). Vgl. hierzu auch Stark, Raymond Aron und der Gestaltwandel des Totalitarismus, S. 195-207.

356 Zur »Machtergreifung«stellte Aron beispielsweise in seinem Beitrag Eine anti-proletarische Revolution. Ideologie und Wirklichkeit des Nationalsozialismus aus dem Jahre 1935 konzis fest: »Hitler hat nicht die Macht ergriffen: man hat sie ihm gegeben, sman<, das sind ein Junker, ein Militär und ein Grundbesitzer aus Ostpreußen.« (Stark, Raymond Aron und der Gestaltwandel des Totalitarismus, S. 167-185, hier S. 184) 
reich und die Demokratien in Europa wahr. Spätestens Mitte des Jahres 1932 war er sich darüber im Klaren, dass sich in Deutschland ein »autoritäres « Regime etablieren würde. ${ }^{357}$ Mit den konkreten politischen Maßnahmen Hitlers nach der Ernennung zum Reichskanzler - Bekämpfung der kommunistischen und sozialdemokratischen Opposition etc. -, seiner »Gleichschaltungspolitik« und speziell dem nationalsozialistischen Antisemitismus beschäftigte sich Aron im September 1933, ohne zu diesem frühen Zeitpunkt das Adjektiv totalitär zur Klassifizierung des NS-Regimes zu verwenden. ${ }^{358}$

Insbesondere im Zusammenhang mit den vom Historiker und Philosophen Elie Halévy Ende 1936 vorgetragenen Thesen zum Zeitalter der Tyranneien nahm Aron allmählich eine totalitarismustheoretische Perspektive ein, indem er den unterschiedlichen politischen, soziologischen und ideengeschichtlichen Ursprüngen der totalitären Bewegungen in Deutschland, Italien und der Sowjetunion nachging. ${ }^{359}$

Während des Zweiten Weltkrieges ging Aron nach London ins Exil, schloss sich der Widerstandsbewegung General de Gaulles an und wurde dort Chefredakteur der demokratischen Zeitschrift La France libre. Nach Kriegsende entschloss er sich, weiterhin journalistisch tätig zu sein, und war vorübergehend Mitherausgeber der Zeitschrift Combat und danach außenpolitischer Mitarbeiter der Pariser Tageszeitung Le Figaro. Daneben veröffentlichte Aron wissenschaftlich-politische Arbeiten, die ihm den Ruf eines (liberal-)konservativen Intellektuellen einbrachten. Im beginnenden Kalten Krieg waren für Aron insbesondere der Stalinismus und die kommunistische Ideologie Gegenstand der kritischen Auseinandersetzung, wiewohl auch das totalitäre NSSystem ihn weiterhin beschäftigte. In einer Sammelrezension ${ }^{360}$ - unter anderem den Studien The Origins of Totalitarianism von Arendts und Bréviaire de la Haine. Le III Reich et les Juifs von Léon Poliakov - untersuchte Aron den »modernen Totalitarismus« und richtete den Fokus insbesondere auf »Ideologie und Terror«, d. h. auf den Zusammen-

357 Raymond Aron, Deutschland, Juni 1932, in: Stark, Raymond Aron und der Gestaltwandel des Totalitarismus, S. 100-110. In seiner Autobiografie beschreibt Aron das gesellschaftliche »Klima des Terrors«. Zu seiner Überraschung stellte er in den Wochen nach dem »]anuar 1933 « fest, dass das »große historische Ereignis« sich quasi geräuschlos vollzog, als wenn sich für die (Berliner) Bevölkerung nichts geändert hätte. Sie machten sozusagen mit. »Es bleibt eine Tatsache, und sie hat uns seinerzeit sehr beeindruckt«, so Aron: »Obwohl die Mehrheit der Wähler sich vor dem Reichstagsbrand und vor dem Verbot der Kommunistischen Partei nie für Hitler und seine Partei ausgesprochen hatte, wurde der Sieg der Nazis vom deutschen Volk akzeptiert.«(Aron, Erkenntnis und Verantwortung, S. 66)

358 Ders., Die nationale Revolution in Deutschland (September 1933), in: Stark, Raymond Aron und der Gestaltwandel des Totalitarismus, S. 141-154.

359 Ders., Das Zeitalter der Tyranneien (Mai 1939), in: Ebd., S. 186-208. Hier benutzte Aron auch schon die Verwendung des Terminus »Politische Religion«, später »säkulare Religion«. Siehe auch den unter dem Eindruck des Hitler-Stalin-Paktes im Juni 1939 verfassten Beitrag Demokratische Staaten und totalitäre Staaten von Aron, in: Ebd., S. 209-241.

360 Raymond Aron, Das Wesen des Totalitarismus (Januar 1954), in: Ebd., S. 275-293. Der französische Originalbeitrag erschien im Januar 1954 in der Zeitschrift Critique, in dem Aron folgende Bücher rezensierte: Arendt, The Origins of Totalitarianism; dies.: Ideologie und Terror, aus: Offener Horizont. Festschrift für Karl Jaspers, hg. von Klaus Piper, München 1953; Léon Poliakov, Bréviaire de la Haine. Le Ille Reich et les Juifs, Paris 1951; Crane Brinton, The Anatomy of Revolution, New York 1952; A. Weissberg, L'Accusé, Paris 1953; F. Beck/W. Godin, Russian Purge and the Extraction of Confession, New York 1951. 
hang von rassistischem Antisemitismus und Judenvernichtung im Nationalsozialismus sowie von Marxismus und Säuberungs- und Terrorphänomen in der Sowjetunion. Wie Arendt war auch Aron der Meinung: »Das Wesen des totalitären Regimes ist der Terror, ein Terror in einem bislang unbekannten Stil. (361 $^{361}$

Nach Le Grand Schisme $e^{362}$ und Les Guerres en Chaine ${ }^{363}$ evozierte vor allem Arons 1955 erschienenes Buch L'opium des intellectuels ${ }^{364}$ eine heftige ideologisch-politische Kontroverse unter den französischen Linksintellektuellen. Insbesondere Jean-Paul Sartre und Maurice Merleau-Ponty waren Zielscheibe von Arons Angriff, »die, bei aller Kritik am Stalinismus, in der Sowjetunion doch die Hoffnung für die Erlösung des >Proletariats s sehen, ein großes Experiment, das trotz allem gewagt werden muß und dessen Irrtümer Nachsicht verdienen, während die Fehler der westlichen Demokratien, allen voran der Vereinigten Staaten, unnachsichtig angeprangert werden müssen « ${ }^{365}$.

Im Monat zählte Aron - wie im französischen Zeitschriftenpendant, der Preuves $\mathrm{zu}$ den frühen Autoren und nutzte das Organ bis zuletzt für zahlreiche Veröffentlichungen. Neben seinem frühen Bemühen für die Integration Deutschlands in das westliche Staatensystem ${ }^{366}$ sowie politischen Reflexionen zum Verhältnis Europas zu den Vereinigten Staaten ${ }^{367}$ und Frankreichs ${ }^{368}$ standen aus zeitgeschichtlichen Gründen die bipolare Weltordnung im Allgemeinen und der (Sowjet-)Kommunismus im Besonderen im Mittelpunkt der Beiträge. Zum Beispiel thematisierte Aron auf dem Berliner "Kongreß für kulturelle Freiheit« in seinem Referat Zwischen dem begrenzten und dem totalen Krieg die internationale politische Situation angesichts der durch die kommunistische Ideologie motivierten expansiven sowjetischen Außenpolitik unter Stalin und warnte - eingedenk der Appeasementpolitik des Westens gegenüber der Außenpolitik Hitlers - vor einer Neutralitätspolitik der demokratischen Staatengemeinschaft und vor einer europäischen »Abkehrpolitik« in Bezug auf die Vereinigten Staaten. Seine Warnung zielte insbesondere in Richtung der westlichen Intellektuellen, angesichts der »totalitären Drohung« und der Gefahr eines »totalen Krieges« sowie von Unzulänglichkeiten in den kapitalistischen demokratischen Staaten Vorstellungen von der >Dritten Kraft anheimzufallen. ${ }^{369}$ Die Faszinationskraft, die die kommunistische Idee (als »weltliche Religion«) und der sowjetische Staat besonders in den Jahren Stalins auf die europäische Linke bzw. europäischen Intellektuellen aus-

361 Ebd., S. 284.

362 Raymond Aron, Le Grand Schisme, Paris 1948.

363 Ders., Les Guerres en Châine, Paris 1951 (dt. Ausg.: Der Permanente Krieg, Frankfurt a. M. 1953).

364 Ders., L'opium des intellectuels, Paris 1955 (dt. Ausg.: Opium für Intellektuelle oder die Sucht nach Weltanschauung, Köln/Berlin 1957).

365 Stark, Raymond Aron und der Cestaltwandel des Totalitarismus, S. 205.

366 Für ein europäisches Deutschland, in: Der Monat 1 (1949), H. 8/9, S. 11-15; Rede vor deutschen Studenten, in: Der Monat 5 (1952), H. 49, S. 3-11.

367 Brief aus Paris: Darf man Kritik üben? Ein französischer Standpunkt (ein Beitrag zum transatlantischen Gespräch), in: Der Monat 5 (1952), H. 50, S. 175-179; Nationale Gesundung und Integration (Aufbau eines neuen Europas), in: Der Monat 5 (1953), H. 54, S. 579-592.

368 Brief aus Paris: Frankreich ohne Minderheit. Haben die Gaullisten de Gaulle besiegt?, in: Der Monat 11 (1959), H. 124, S. 69-75; de Gaulles Alleingang, in: Der Monat 12 (1960), H. 139, S. 14-22.

369 Zwischen dem begrenzten und dem totalen Krieg, in: Der Monat 2 (1950), H. 22/23, S. 455-462. Siehe ausführlich Raymond Aron, Der Permanente Krieg, Frankfurt a. M. 1953. Das letzte Kapitel des Buches mit dem Titel Glaube ohne Illusionen wurde im Monat veröffentlicht (5 [1953], H. 60, S. 563-571). 
übte, beleuchtete Aron in Derfalsche Messias. ${ }^{370}$ In seinem letzten Beitrag für den Monat mit dem Titel Noch einmal: Hitler. Wie haltbar ist die Totalitarismus-Theorie untersuchte Aron den Nationalsozialismus unter explizit totalitarismustheoretischer Perspektive und verglich in diesem Zusammenhang die Verlaufsform der »roten und braunen Revolution ${ }^{371}$

Aron hat nach Kriegsende ein umfangreiches, facettenreiches Gesamtwerk hinterlassen, das neben dem Nachdenken über das Totalitarismusphänomen unter anderem die Themenbereiche Soziologie, ${ }^{372}$ internationale Beziehungen, ${ }^{373}$ Ideologiekritik, ${ }^{374}$ (Welt-)Politik ${ }^{375}$ und politische Theorie umfasste. Unter dem Einfluss von Tocqueville stehend, bestand das primäre Ziel von Arons Reflexionen in der Legitimation von politischer Freiheit. ${ }^{376}$ Neben verschiedenen Lehrtätigkeiten ab 1945 - z. B. als Soziologieprofessor an der Pariser Sorbonne - war Aron journalistisch tätig. Von 1947 bis 1977 schrieb er im Figaro wöchentlich Kommentare zur internationalen Politik und fungierte danach bis zu seinem Tode im Oktober 1983 als Herausgeber und Kolumnist für das Wochenmagazin L'Express.

\subsection{Hans Kohn}

$\mathrm{Zu}$ einem der wichtigsten Autoren des Monat entwickelte sich der jüdische Historiker und Nationalismusforscher Hans $\mathrm{Kohn}^{377}$, der sich nicht zuletzt aufgrund seiner individuellen Erfahrungen in seinen zahlreichen Veröffentlichungen mit den ideen- und gesellschaftsgeschichtlichen Ursprüngen der politischen und geistigen Erschütterun-

370 Der falsche Messias. Ein Schlußwort zu »The God that failed«, in: Der Monat 3 (1950), H. 26, S. 175-184 (hierbei handelte es sich um das Nachwort für die französische Ausgabe von Richard H. S. Crossmanns Sammelband The God that failed, das unter dem Titel Le Dieu des Ténèbres 1950 in Paris erschien).

371 Noch einmal: Hitler. Wie haltbar ist die Totalitarismus-Theorie?, in: Der Monat 278 (1981), H. 1, S. 4255. Eine längere Fassung erschien unter dem Titel Gibtes ein Nazi-Rätsel in: Stark, Raymond Aron und der Gestaltwandel des Totalitarismus, S. 294-320.

372 Siehe u. a.: La societé industrielle et la guerre, Paris 1958 sowie Dix-huit leçons sur la societé, Paris 1962 (dt. Ausg.: Die industrielle Gesellschaft, Frankfurt a. M. 1964)

373 Siehe u. a.: Paix et guerre entre les Nations, Paris 1961 (dt. Ausg.: Frieden und Krieg. Eine Theorie der Staatenwelt, Frankfurt a. M. 1963).

374 Siehe u. a.: Marxismes imaginaires. D’une famille à l'autre, Paris 1970 (dt. Ausg.: Die heiligen Familien des Marxismus, Hamburg 1970) sowie Les Dèsillusions du progrès, Paris 1969 (dt. Ausg. Fortschritt ohne Ende. Über die Zukunft der Industriegesellschaft, München 1970).

375 Siehe u. a.: La tragédie algérienne, Paris 1957; L’Algérie et la République, Paris 1958; République impériale, les Etats-Unis dans le monde 1945-1972, Paris 1973 (dt. Ausg.: Die imperiale Republik: die Vereinigten Staaten von Amerika und die übrige Welt seit 1945, Stuttgart 1975); Plaidoyer pour l'Europe dècadente, Paris 1977 (dt. Ausg.: Plädoyer für das dekadente Europa); La Révolution introuvable. Réflexions sur la Révolution de Mai, Paris 1968.

376 Siehe hierzu bes.: Essai sur les libertés, Paris 1965 (dt. Ausg.: Über die Freiheiten. Essay, Stuttgart 1981) sowie Démocratie et totalitarisme, Paris 1965 (dt. Ausg.: Demokratie und Totalitarismus, Hamburg 1970).

377 Bevor Kohn als Autor für den Monat in Erscheinung trat, veröffentlichte er - wie auch beispielsweise Arendt - bereits in der ab 1945 (bis 1949) von Dolf Sternberger - unter Mitwirkung von Karl Jaspers, Werner Kraus und Alfred Weber-herausgegebenen Monatszeitschrift Die Wandlung. 